International Journal of Pure and Applied Mathematics

Volume 89 No. 2 2013, 203-213

ISSN: 1311-8080 (printed version); ISSN: 1314-3395 (on-line version)

url: http://www.ijpam.eu

doi: http://dx.doi.org/10.12732/ijpam.v89i2.6

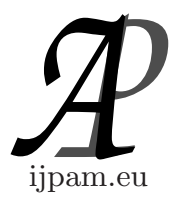

\title{
ERRORS AND GRIDS FOR PROJECTED WEAKLY SINGULAR INTEGRAL EQUATIONS
}

\author{
F.D. d'Almeida ${ }^{1}$, M. Ahues ${ }^{2}$, R. Fernandes ${ }^{3} \S$ \\ ${ }^{1}$ Centro de Matemática and Faculdade de Engenharia \\ da Universidade Porto (CMUP) \\ Rua Roberto Frias, 4200-465 Porto, PORTUGAL \\ ${ }^{2}$ Institut Camille Jordan \\ Université Jean Monnet \\ Membre d'Université de Lyon \\ 23 rue Dr Paul Michelon, 42023 St-Étienne, FRANCE \\ ${ }^{3}$ Centro de Matemática and Departamento \\ de Matemática e Aplicações \\ da Universidade do Minho \\ Campus de Gualtar, 4710-057 Braga, PORTUGAL
}

\begin{abstract}
In the solution of weakly singular Fredholm integral equations of the second kind defined on the space of Lebesgue integrable complex valued functions by projection methods, the choice of the grid is crucial. We will present the proof of an error bound in terms of the mesh size of the underlying discretization grid on which no regularity assumptions are made and compare it with other recently proposed error bounds. This proof generalizes the work done for the Galerkin method, to the case of Kantorovich and Sloan methods. This allows us to use nonuniform grids when there are boundary layers or discontinuities in the right hand side of the equation. We illustrate this with an example on the radiative transfer model in stellar atmospheres.
\end{abstract}

AMS Subject Classification: 65J10, 65R20

Received: August 23, 2013

(C) 2013 Academic Publications, Ltd. url: www.acadpubl.eu

${ }^{\S}$ Correspondence author 
Key Words: projection approximations in $L^{1}$, weakly singular integral operators, nonuniform grids, error bounds

\section{Introduction}

We consider the Fredholm integral equation of the second kind

$$
(T-z I) \varphi=f,
$$

where $T: X \rightarrow X$ is a linear compact integral operator, $X$ is a Banach space, $z$ is in the resolvent set, $\operatorname{re}(T)$, and hence $z \neq 0$ since $T$ is compact. For each $f \in X$, equation (1) has a unique solution $\varphi \in X$.

Let $X:=L^{1}\left(\left[0, \tau^{*}\right], \mathbb{C}\right)$ be the space of complex-valued Lebesgue-integrable (classes of) functions on a closed interval $\left[0, \tau^{*}\right] . T$ is the operator defined on $X$ by

$$
(T x)(s):=\int_{0}^{\tau^{*}} g(|s-t|) x(t) d t, s \in\left[0, \tau^{*}\right],
$$

where $g:] 0,+\infty[\rightarrow \mathbb{R}$ is a weakly singular function at 0 in the sense that

$$
g\left(0^{+}\right)=+\infty, g \in L^{1}\left(\left[0,+\infty[, \mathbb{R}) \cap C^{0}(] 0,+\infty[, \mathbb{R}) .\right.\right.
$$

To make technical aspects simpler, we also assume that in ]0, $+\infty[$,

$$
g \text { is a nonnegative decreasing function. }
$$

Since the solution $\varphi$ of (1) satisfies

$$
\varphi=\frac{1}{z}(T \varphi-f),
$$

we may expect boundary layers for $\varphi$ at the end points of the domain and where $f$ behaves in a similar way. For details, see [4]. For this reason, the possibility of using nonuniform grids, thus allowing for better refinement in the sensitive areas, is important.

\section{Projection Approximations}

Let us consider a sequence $\left(\pi_{n}\right)_{n \geq 1}$ of bounded projections each one having finite rank and range $X_{n} \subset X$. Then, the classical projection approximation methods for the solution of (1) use the following operators: $T_{n}^{G}:=\pi_{n} T \pi_{n}, T_{n}^{K}:=\pi_{n} T$, 
$T_{n}^{S}:=T \pi_{n}$, where the upper label $G$ refers to the Galerkin method, $K$ to the Kantorovich method and $S$ to the Sloan method. Each $T_{n} \in\left\{T_{n}^{G}, T_{n}^{K}, T_{n}^{S}\right\}$ is a bounded linear operator, and $\left(T_{n}\right)_{n \geq 1}$ is, at least, $\nu$-convergent to $T$, meaning that $\left(\left\|T_{n}\right\|\right)_{n \geq 1}$ is bounded, $\left\|\left(T_{n}-T\right) T\right\| \rightarrow 0$, and $\left\|\left(T_{n}-T\right) T_{n}\right\| \rightarrow 0$, (see [6]). In the case of Kantorovich method, the convergence is uniform. We use one of these approximate operators to set an approximate problem

$$
\left(T_{n}-z I\right) \varphi_{n}=f
$$

(or $\left(T_{n}-z I\right) \varphi_{n}=\pi_{n} f$, in the case of the Galerkin approximation). It is known that if $z \in \operatorname{re}(T)$, then, for $n$ large enough, $z \in \operatorname{re}\left(T_{n}\right)$ and $\varphi_{n}=\left(T_{n}-z I\right)^{-1} f$.

Theorem 1. Let $\left(\pi_{n}\right)_{n \geq 1}$ be a sequence of projections onto $X_{n}$, pointwise convergent to $I$. Then there exists $n_{0}$ such that for all $n \geq n_{0}$,

$$
\begin{aligned}
\left\|\varphi_{n}^{G}-\varphi\right\| & \leq \beta^{G}\left\|\left(I-\pi_{n}\right) \varphi\right\|, \\
\left\|\varphi_{n}^{K}-\varphi\right\| & \leq \beta^{K}\left(\left\|\left(I-\pi_{n}\right) \varphi\right\|+\frac{1}{|z|}\left\|\left(I-\pi_{n}\right) f\right\|\right), \\
\left\|\varphi_{n}^{S}-\varphi\right\| & \leq \beta^{S}\left\|\left(I-\pi_{n}\right) \varphi\right\|,
\end{aligned}
$$

where the constants

$$
\begin{aligned}
\beta^{G} & :=|z| \sup _{n \geq n_{0}}\left\|\left(\pi_{n} T-z I\right)^{-1}\right\|, \\
\beta^{K} & :=|z| \sup _{n \geq n_{0}}\left\|\left(\pi_{n} T-z I\right)^{-1}\right\|, \\
\beta^{S} & :=\|T\| \sup _{n \geq n_{0}}\left\|\left(T \pi_{n}-z I\right)^{-1}\right\|,
\end{aligned}
$$

are finite.

Proof. We will prove the inequality for the Kantorovich and Sloan cases, since the case of the Galerkin method was addressed in [4]. The constants $\beta^{K}$ and $\beta^{S}$ are finite due to the compactness of $T$ and consequent convergence of $\left(\pi_{n} T\right)_{n \geq 1}$ in norm to $T$, in the Kantorovich case, or in the $v$-convergence sense, in the Sloan method (see [6] and [7]). Let us consider the following equalities based on equation (4), its projection by $\pi_{n}$ and the approximate problem (5), corresponding to Kantorovich or Sloan method,

$$
\begin{aligned}
\varphi & =\frac{1}{z}(T \varphi-f), \pi_{n} \varphi=\frac{1}{z}\left(\pi_{n} T \varphi-\pi_{n} f\right), \\
\varphi_{n}^{K} & =\frac{1}{z}\left(\pi_{n} T \varphi_{n}^{K}-f\right), \varphi_{n}^{S}=\frac{1}{z}\left(T \pi_{n} \varphi_{n}^{S}-f\right),
\end{aligned}
$$


then, for the Kantorovich case, we have

$$
\begin{aligned}
\varphi-\pi_{n} \varphi & =\varphi-\varphi_{n}^{K}+\varphi_{n}^{K}-\pi_{n} \varphi \\
& =\frac{1}{z}\left(\left(\pi_{n} T-z I\right)\left(\varphi_{n}^{K}-\varphi\right)-\left(I-\pi_{n}\right) f\right)
\end{aligned}
$$

and

$$
\varphi_{n}^{K}-\varphi=z\left(\pi_{n} T-z I\right)^{-1}\left(\left(I-\pi_{n}\right) \varphi+\frac{1}{z}\left(I-\pi_{n}\right) f\right) .
$$

Now, let us consider the case of the Sloan method:

$$
\begin{aligned}
\varphi-\pi_{n} \varphi & =\varphi-\varphi_{n}^{S}+\varphi_{n}^{S}-\pi_{n} \varphi \\
& =\frac{1}{z}\left(T \pi_{n}-z I\right)\left(\varphi_{n}^{S}-\varphi\right)-\frac{1}{z}(T-z I)\left(I-\pi_{n}\right) \varphi
\end{aligned}
$$

and so $\varphi_{n}^{S}-\varphi=\left(T \pi_{n}-z I\right)^{-1} T\left(I-\pi_{n}\right) \varphi$. Hence

$$
\left\|\varphi_{n}^{S}-\varphi\right\| \leq\|T\|\left\|\left(T \pi_{n}-z I\right)^{-1}\right\|\left\|\left(I-\pi_{n}\right) \varphi\right\| \leq \beta^{S}\left\|\left(I-\pi_{n}\right) \varphi\right\| .
$$

This concludes the proof.

\section{Discretization Grids and Error Bounds}

Let us consider a general grid $\mathcal{G}_{n}:=\left(\tau_{j}\right)_{j=0}^{n}$ set on $\left[0, \tau^{*}\right]$ such that

$$
\begin{gathered}
\tau_{0}:=0, \tau_{n}:=\tau^{*}, h_{j}:=\tau_{j}-\tau_{j-1}>0, \\
h_{\max }:=\max _{1 \leq j \leq n} h_{j} \text { and } h_{\min }:=\min _{1 \leq j \leq n} h_{j} .
\end{gathered}
$$

We associate to this grid the local mean functionals $e_{j}^{*}$ defined by

$$
\left\langle x, e_{j}^{*}\right\rangle:=\frac{1}{h_{j}} \int_{\tau_{j-1}}^{\tau_{j}} x(t) d t,
$$

and the piecewise constant canonical functions $e_{j}$ given by

$$
e_{j}(s):= \begin{cases}1 & \text { for } s \in\left[\tau_{j-1}, \tau_{j}\right] \\ 0 & \text { otherwise }\end{cases}
$$


We define the projections onto the subspace $X_{n}$, spanned by $\left\{e_{j}, j=1, \ldots, n\right\}$, as

$$
\pi_{n} x:=\sum_{j=1}^{n}\left\langle x, e_{j}^{*}\right\rangle e_{j} \quad, \text { for } x \in L^{1}\left(\left[0, \tau^{*}\right], \mathbb{C}\right) .
$$

In order to estimate the relative error of the Kantorovich and the Sloan approximations, in terms of the grid parameters, mainly $h_{\max }$, we have the following theorem:

Theorem 2. The relative error of Kantorovich and Sloan approximations satisfy:

$$
\begin{gathered}
\frac{\left\|\varphi_{n}^{K}-\varphi\right\|}{\|\varphi\|} \leq 8 C^{K} \int_{0}^{h_{\max } / 2} g(\tau) d \tau \\
\frac{\left\|\varphi_{n}^{S}-\varphi\right\|}{\|\varphi\|} \leq C^{S}\left[8 \int_{0}^{h_{\max } / 2} g(\tau) d \tau+\frac{2}{\|\varphi\|} \sum_{j=1}^{n} \omega_{1}\left(f_{\mid\left[\tau_{j-1}, \tau_{j}\right]}, h_{j}\right)\right]
\end{gathered}
$$

where, see [9],

$$
\begin{gathered}
\omega_{1}\left(x_{\mid[a, b]}, \delta\right):=\sup _{0 \leq h \leq \delta} \int_{a}^{b-h}|x(s+h)-x(s)| d s \\
C^{K}:=\sup _{n \geq n_{0}}\left\|\left(\pi_{n} T-z I\right)^{-1}\right\|, C^{S}:=\frac{\|T\|}{|z|} \sup _{n \geq n_{0}}\left\|\left(T \pi_{n}-z I\right)^{-1}\right\| .
\end{gathered}
$$

Proof. From (4) and (6), we have

$$
\begin{aligned}
\varphi_{n}^{K}-\varphi & =\left(\pi_{n} T-z I\right)^{-1}\left(I-\pi_{n}\right)(z \varphi+f) \\
& =\left(\pi_{n} T-z I\right)^{-1}\left(\left(I-\pi_{n}\right) T \varphi\right)
\end{aligned}
$$

hence

$$
\frac{\left\|\varphi_{n}^{K}-\varphi\right\|}{\|\varphi\|} \leq \sup _{n \geq n_{0}}\left\|\left(\pi_{n} T-z I\right)^{-1}\right\|\left\|\left(I-\pi_{n}\right) T\right\|
$$

The fact that $\left\|\left(I-\pi_{n}\right) T\right\| \leq 8 \int_{0}^{h_{\max } / 2} g(\tau) d \tau$ is proved in [4]. Similarly, for the Sloan approximation, $\varphi_{n}^{S}$, we have to bound

$$
\left\|\varphi_{n}^{S}-\varphi\right\| \leq\|T\| \sup _{n \geq n_{0}}\left\|\left(T \pi_{n}-z I\right)^{-1}\right\|\left\|\left(I-\pi_{n}\right) \varphi\right\| .
$$


Using equation (1) we have

$$
\left(I-\pi_{n}\right) \varphi=\frac{1}{z}\left(I-\pi_{n}\right)(T \varphi-f)
$$

hence

$$
\left\|\left(I-\pi_{n}\right) \varphi\right\|=\frac{1}{|z|}\left(\left\|\left(I-\pi_{n}\right) T\right\|\|\varphi\|+\left\|\left(I-\pi_{n}\right) f\right\|\right) .
$$

Here again we refer to [4] to conclude that

which ends the proof.

$$
\left\|\left(I-\pi_{n}\right) T\right\| \leq 8 \int_{0}^{h_{\max } / 2} g(\tau) d \tau, \quad\left\|\left(I-\pi_{n}\right) f\right\| \leq 2 \sum_{j=1}^{n} \omega_{1}\left(f_{\mid\left[\tau_{j-1}, \tau_{j}\right]}, h_{j}\right)
$$

The error bounds given in this theorem will be compared to the following ones, on an example, in the next section. In [5], we find

$$
\begin{aligned}
& \left\|\left(I-\pi_{n}\right) T\right\| \leq 2 h_{\max }\left(g\left(h_{\min } / 2\right)-g\left(\tau^{*}\right)\right) \\
& +2 h_{\max }\left(g\left(h_{\min }\right)-g\left(\tau^{*}\right)\right)+4 \int_{0}^{h_{\max } / 2} g(\sigma) d \sigma \\
& +4 \int_{0}^{h_{\max }} g(\sigma) d \sigma+4 \int_{0}^{3 h_{\max } / 2} g(\sigma) d \sigma .
\end{aligned}
$$

Although this is less sharp than the one proposed here, it may be interesting since it is set in terms of the maximum and minimum values of the amplitudes of the subintervals, and its proof is based on geometric considerations. And in $[2]$, we find

$$
\left\|\left(I-\pi_{n}\right) T\right\| \leq 4\left[\int_{0}^{h_{\max } / 2} g(\sigma) d \sigma-h_{\max } \int_{h_{\max } / 2}^{+\infty} g^{\prime}(\sigma) d \sigma\right],
$$

which requires the piecewise derivability of the kernel.

\section{Numerical Computations}

The computations that we will show were done with an integral operator that comes from a simplified model of radiative transfer in stellar atmospheres. Its kernel is $g(s):=\frac{\varpi}{2} E_{1}(s)$, where $E_{1}$ is the first exponential integral function (see $[1]): E_{1}(s):=\int_{0}^{1} \frac{\exp (-s / \mu)}{\mu} d \mu$, and $\left.\left.s \in\right] 0, \tau^{*}\right]$ represents the optical depth of the 
stellar atmosphere and $\left.\tau^{*} \in\right] 0,+\infty[$ the optical thickness. The albedo $\varpi \in] 0,1[$ measures the scattering properties of the medium. Here $z=1, \tau^{*}=100$, $\varpi=0.75$ and $f$ in (1) is

$$
f(s):=\left\{\begin{array}{rll}
-1 & \text { for } \quad 0 \leq s \leq 50 \\
0 & \text { for } \quad 50<s \leq 100 .
\end{array}\right.
$$

For details see [3] and [8].

The grids on $[0,100]$ for this example are two uniform grids and two nonuniform ones $(n=500, n=1000)$ as described in Table 1. Computations have been performed with Matlab. We have computed the relative error of the approximations with respect to a reference solution, $\varphi^{\text {ref }}$ (see Fig. 1), obtained with a grid of 4001 points.

\begin{tabular}{|c|c||c|c|c|c|c|}
\hline Subintervals & Nonuniform & \multicolumn{5}{|c|}{ Zones } \\
\cline { 3 - 7 }$n$ & grids & {$[0,10]$} & {$[10,40]$} & {$[40,60]$} & {$[60,90]$} & {$[90,100]$} \\
\hline 500 & Subintervals & 170 & 25 & 280 & 10 & 15 \\
\cline { 3 - 7 } & per zone & 340 & 50 & 560 & 20 & 30 \\
\hline 1000 & & &
\end{tabular}

Table 1: Nonuniform grids in 5 zones of $[0,100]$

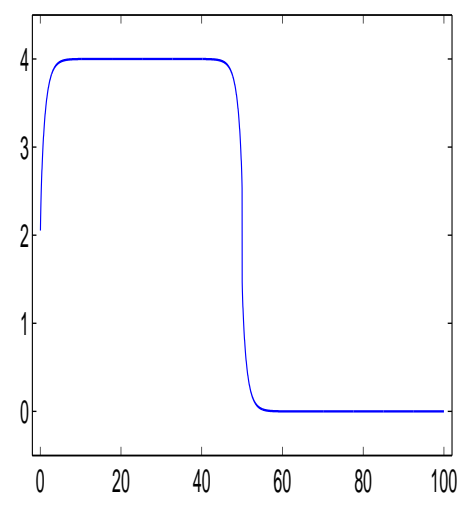

Figure 1: Reference solution $\varphi^{\text {ref }}$

In Fig. 2 we compare the error of $\varphi_{500}^{K}$ obtained with 500 equal subintervals with the error of $\varphi_{1000}^{K}$ obtained with 1000 equal subintervals. The error is reduced by one half, as expected.

If we distribute the 501 points in a nonuniform grid as in Table 1, thus refining the grid more in the Zone 1 where a boundary layer is expected, due 


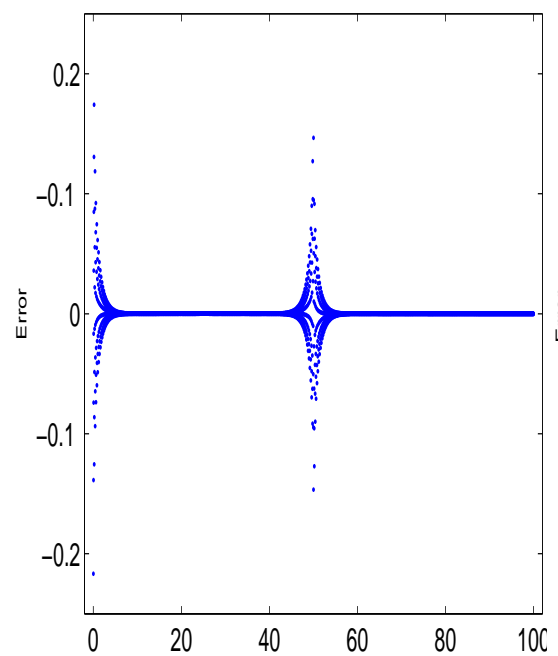

(a) Uniform: $\mathrm{n}=500$

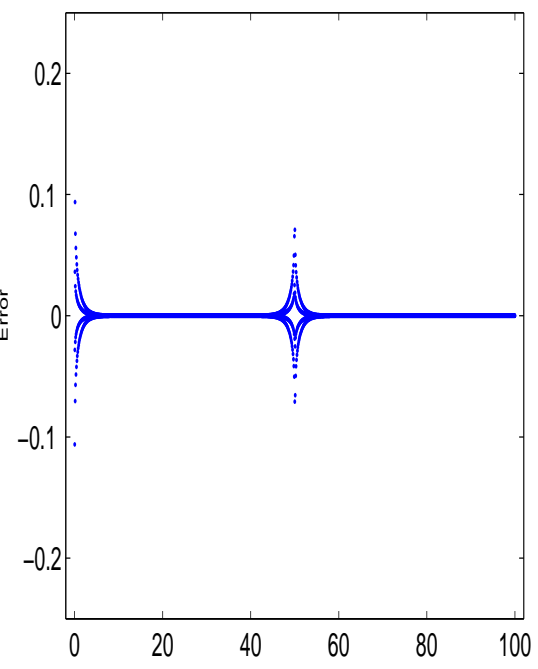

(b) Uniform: $\mathrm{n}=1000$

Figure 2: $\varphi^{\text {ref }}-\varphi_{n}^{K}$ with uniform grids

to the singularity of the kernel, and in the middle of the interval where $f$ has a discontinuity, and setting large subintervals in the other zones, we get an overall error that is smaller than the error of the solution $\varphi_{1000}^{K}$ obtained with a uniform grid twice finer, as it is shown in Fig. 3.

\begin{tabular}{|c|c|c|c|c|c|}
\hline \multirow[t]{2}{*}{$n$} & \multirow[t]{2}{*}{ Grid } & \multicolumn{3}{|c|}{ Error bound } & \multirow{2}{*}{$\begin{array}{l}\text { Relative } \\
\text { error }\end{array}$} \\
\hline & & EB1 & EB2 & EB3 & \\
\hline 500 & $\begin{array}{c}\text { Uniform } \\
h_{\max }=1 / 5\end{array}$ & $3.3 \mathrm{E}+0$ & $9.3 \mathrm{E}+0$ & $3.9 \mathrm{E}+0$ & $1.2 \mathrm{E}-3$ \\
\hline 500 & $\begin{array}{c}\text { Nonuniform } \\
h_{\min }=1 / 17 \\
h_{\max }=3\end{array}$ & $1.2 \mathrm{E}+1$ & $6.6 \mathrm{E}+1$ & $7.4 \mathrm{E}+0$ & $4.6 \mathrm{E}-4$ \\
\hline 1000 & $\begin{array}{c}\text { Uniform } \\
h_{\max }=1 / 10\end{array}$ & $2.1 \mathrm{E}+0$ & $6.2 \mathrm{E}+0$ & $2.6 \mathrm{E}+0$ & $6.3 \mathrm{E}-4$ \\
\hline 1000 & $\begin{array}{c}\text { Nonuniform } \\
h_{\min }=1 / 34 \\
h_{\max }=3 / 2\end{array}$ & $9.4 \mathrm{E}+0$ & $4.6 \mathrm{E}+1$ & $7.8 \mathrm{E}+0$ & $3.0 \mathrm{E}-4$ \\
\hline
\end{tabular}

Table 2: $L^{1}$-Errors of the Kantorovich approximation

The computation of the three error bounds referred in this work for the Kantorovich approximation and this example, yields the values presented in Table 2, for uniform and nonuniform grids. The error bounds will be denoted 


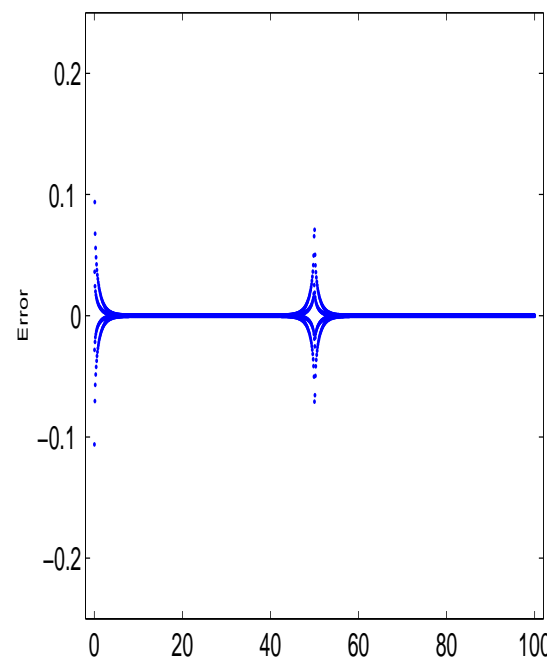

(a) Uniform: $\mathrm{n}=1000$

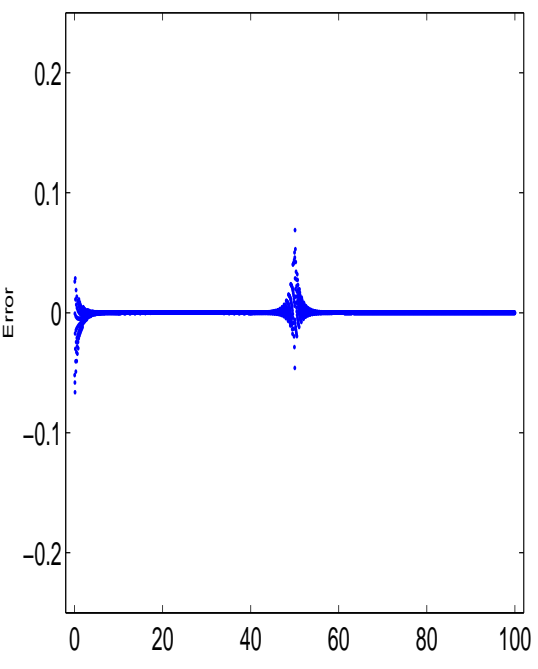

(b) Nonuniform: $\mathrm{n}=500$

Figure 3: $\varphi^{\text {ref }}-\varphi_{n}^{K}$ with uniform and nonuniform grids

by EB1, corresponding to Eq.(7), EB2, corresponding to Eq.(8) and (10) and EB3 corresponding to Eq.(8) and (11). This table also contains the $L^{1}-$ norm of the a posteriori relative error, with respect to the reference solution $\varphi^{r e f}$.

As we can see all the three error bounds are pessimistic in the sense that they are much worse than the relative error. However they do not require the grid to be uniform.

Comparing the three ways of computing the error bounds we can see that EB1 is the best for the case of uniform grids. In the case of nonuniform grids the best is the bound given by M. Ahues, A. Amosov, A. Largillier in [2] but it requires the piecewise derivability of the kernel.

\section{Conclusions}

In this work we give a proof of an error bound for the approximate solutions of weakly singular Fredholm integral equations, that generalizes the work presented in [4] for the Galerkin method to the case of Kantorovich and Sloan mehtods. It is set in terms of the mesh size of the underlying discretization 
grid.

This error bound is compared with other error bounds proposed in [5] and [2] on an example issued from the radiative transfer modelling in stellar atmospheres, and proved to be the best for uniform grids.

The use of nonuniform grids is addressed as they allow the refinement of grids, where there are boundary layers or discontinuities in the right hand side of the equation, without increasing the overall number of subintervals. The present bound does not require the grids to be uniform, and although in the example given it does not perform so well as the one in [2], it does not require the piecewise derivability of the kernel.

\section{Acknowledgments}

Financial support provided by the Research Centre of Mathematics of the University of Minho through the FCT Pluriannual Funding Program, and through CMUP, by the European Regional Development Fund through the programme COMPETE and by the Portuguese Government through the FCT - Fundação para a Ciência e a Tecnologia under the project PEst-C/MAT/UI0144/2011.

\section{References}

[1] M. Abramowitz, I.A. Stegun, Handbook of Mathematical Functions, Dover Publications, New York (1972).

[2] M. Ahues, A. Amosov, A. Largillier, Superconvergence of some projection approximations for weakly singular integral equations using general grids, SIAM J. Numer. Anal., 47, No. 1 (2009), 646-674. DOI: $10.1137 / 070685464$

[3] M. Ahues, F. D. d'Almeida, A. Largillier, O. Titaud, P. B. Vasconcelos, An $\mathrm{L}^{1}$ Refined Projection Approximate Solution of the Radiation Transfer Equation in Stellar Atmospheres, Journal of Computational and Applied Mathematics, 140 (2002), 13-26. DOI: 10.1016/S0377-0427(01)00403-4

[4] M. Ahues, F. d' Almeida, R. Fernandes, Piecewise constant Galerkin approximations of weakly singular integral equations, Internat. J. Pure Appl. Math, 55, No. 4 (2009), 569-580. 
[5] M. Ahues, F.D. d' Almeida, R. Fernandes, Error Bounds for $L^{1}$ Galerkin Approximations of Weakly Singular Integral Operators, In: Integral Methods in Science and Engineering, volume 2: Computational Methods, Birkäuser, (2010), 1-10. DOI: 10.1007/978-0-8176-4897-8_1

[6] M. Ahues, A. Largillier, B. V. Limaye, Spectral Computations with Bounded Operators, CRC, Boca Raton (2001).

[7] K. Atkinson, The numerical solution of integral equations of the second kind, n. 4 in Cambridge Monographs on Applied and Computational Mathematics, Cambridge University Press (1997).

[8] F. D. d' Almeida, P. B. Vasconcelos, R. Fernandes, Discretization of Iterative Refinement Methods for a Transfer Integral Equation, In Proceedings of the International Conference on Topics in Functional and Numerical Analysis (TOFNA-2005), J. Analysis, 14 (2006), 33-53.

[9] A.V. Efimov, Modulus of Continuity, In Encyclopæedia of Mathematics, Springer (2001). 
